Eur Biophys J (1989) 17: 233-235

\title{
Effect of aminoacylation on tRNA conformation
}

\author{
J. Antosiewicz * and D. Porschke \\ Max-Planck-Institut für biophysikalische Chemie, Am Fassberg, D-3400 Göttingen, Federal Republic of Germany
}

Received June 8, 1989/Accepted in revised form August 3, 1989

\begin{abstract}
Translational diffusion coefficients have been simulated for various conformations of tRNA $\mathrm{Ah}^{\text {Phe }}$ (yeast) by bead models, in order to analyze data obtained by dynamic light scattering on the free and the aminoacylated form. The $18 \%$ increase of the translational diffusion coefficient upon deacylation, reported by Potts et al. (1981), could not be represented by any change of the L-hinge angle, but could only be simulated by a conformation change to an extended form with extensive dissociation of base pairs. Since extensive unpairing is not consistent with evidence accumulated in the literature, the change of the diffusion coefficient must be mainly due to processes other than intramolecular conformational changes.
\end{abstract}

Key words: Bead model simulation of tRNA, tRNAaminoacylation, tRNA-conformation, tRNA-bead model simulation, tRNA-translation diffusion coefficient

\section{Introduction}

The recent detection of catalytic activities (Cech 1987) of RNA's has raised the general interest in the potential of these molecules. Among the exceptional effects reported for RNA's is the conformation change of tRNA $^{\text {Phe }}$ upon aminoacylation (Potts et al. 1977; Davanloo et al. 1979; Potts et al. 1981), which is of great interest for protein-biosynthesis. It is generally accepted that the strongest evidence for such a conformation change comes from the measurements of laser light scattering by Potts et al. (1981), who reported an increase of the translational diffusion coefficient upon deacylation by $18 \%$. We have tried to interpret these data quantitatively by bead model simulations.

Bead models can be used to simulate translational (and rotational) diffusion coefficients for objects of

* Present address: Institute of Experimental Physics, Department of Biophysics, Warsaw University, PL-02-089 Warsaw, Poland complex shape, which cannot be represented by spheres, ellipsoids or cylinders. The technique has been described in detail and tested extensively (Brenner 1967; Harvey and Garcia de la Torre 1980; Garcia de la Torre and Bloomfield 1981). We apply the procedure developed for "overlapping" beads, which requires models constructed from beads of uniform size.

\section{Results and discussion}

For our bead model simulations we start from the known x-ray structure (Ladner et al. 1975; Quigley et al. 1975) and substitute each nucleotide by a bead. The center of each bead corresponds to the center of mass, which is calculated for each nucleotide assuming equal mass for the constituting atoms. An initial value of the bead radius may be derived from the known $\mathrm{x}$-ray structure; fine tuning is possible by comparison with experimental data for translational or rotational diffusion. When we use data for rotational diffusion (in preparation), we arrive at a bead radius of approximately $6.4 \AA$. However, a model constructed with this bead radius leads to a translational diffusion coefficient of $8.22 \cdot 10^{7} \mathrm{~cm} \mathrm{~s}^{-1}$, which is clearly larger than the value reported by Potts et al. (1981) in a buffer (0.1 M Tris- $\mathrm{HCl}, \mathrm{pH} 7.2,0.1 \mathrm{M} \mathrm{NaCl}, 10 \mathrm{mM} \mathrm{MgCl}$ ), where the maximal changes upon aminoacylation have been observed. We can easily adjust the bead radius of our model to fit the $\mathrm{D}_{t}$ value of Potts et al. (1981) and then arrive at a bead radius of $10.4 \AA$. As shown by the simulations described below and the data compiled in Table 1, we do not have to model the diffusion coefficients absolutely. For our purpose it is sufficient to model the relative change of the diffusion coefficient, which is not very much dependent on the bead size in the range from $6.4 \AA$ to $10.4 \AA$.

We start our simulation of the data on the aminoacylation by adding a bead to the CCA end, which reflects the attached amino acid. We are aware of the 

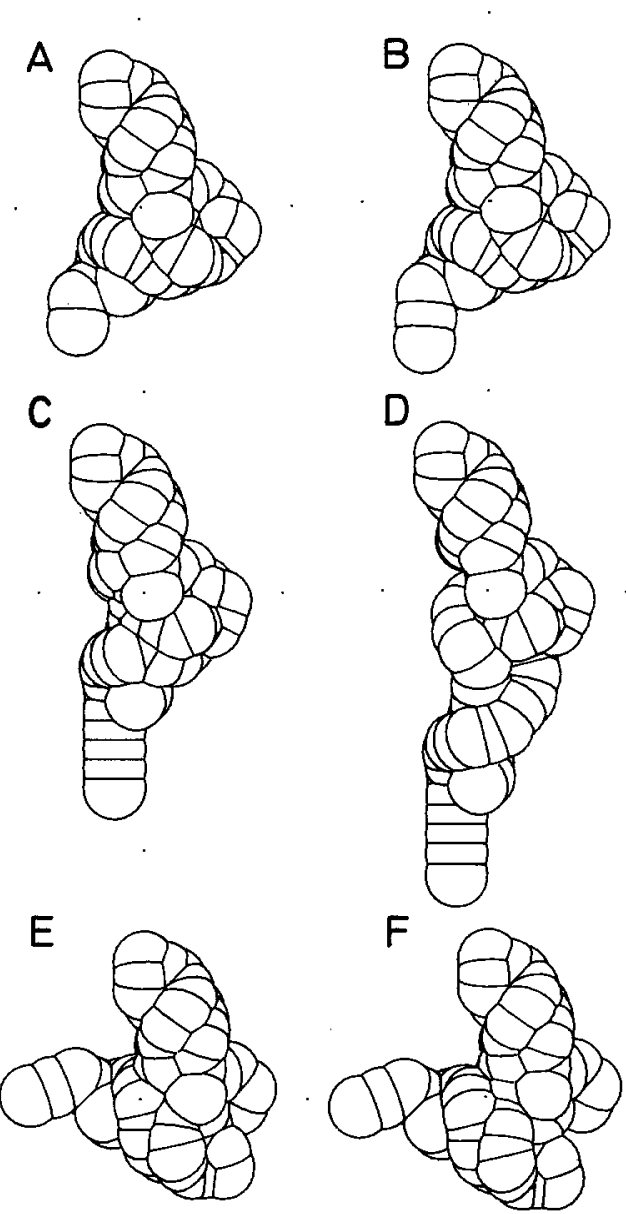

Fig. 1. Bead models of tRNA ${ }^{\text {Phe }}$ with a bead radius of $10.4 \AA$. A Crystal structure; $B$ bead added at CCA end to simulate amino acid; $\mathbf{C}$ same as $\mathbf{B}$, but elongated by change of the L-hinge angle and of the CCA end; $\mathbf{D}$ same as $\mathbf{C}$, but more elongated by disruption of secondary and tertiary structure; $\mathbf{E}$ form generated by an decrease of the L-hinge angle; $\mathbf{F}$ same as $\mathbf{E}$, but partly unfolded

Table 1. Translational diffusion coefficients (in units of $10^{-7} \mathrm{~cm}^{2}$ $\mathrm{s}^{-1}$ ) simulated for bead models of tRNA ${ }^{\text {Phe }}$ structures (cf. Fig. 1 and text) with two different values of the bead radius. The $\Delta-$ values are the changes of $D_{t}$ relative to the $D_{t}$-value of model $A$ in \%-units. The models $\mathrm{A}$ to $\mathrm{F}$ correspond to those shown in Fig. 1; model G corresponds so that shown in Fig. $1 \mathrm{E}$, but without the terminal bead representing the amino acid

\begin{tabular}{|c|c|c|c|c|}
\hline \multirow[t]{3}{*}{ Model } & \multicolumn{4}{|c|}{ Bead radius } \\
\hline & \multicolumn{2}{|c|}{$6.4 \AA$} & \multicolumn{2}{|c|}{$10.4 \AA$} \\
\hline & $\mathrm{D}_{t}$ & $\Delta[\%]$ & $\mathrm{D}_{t}$ & $\Delta[\%]$ \\
\hline A & 8.22 & 0.0 & 7.06 & 0.0 \\
\hline B & 7.99 & 2.80 & 6.87 & 2.69 \\
\hline C & 7.56 & 8.03 & 6.51 & 7.79 \\
\hline $\mathrm{D}$ & 6.70 & 18.5 & 5.84 & 17.3 \\
\hline $\mathrm{E}$ & 7.95 & 3.28 & 6.84 & 3.12 \\
\hline $\mathrm{F}$ & 7.65 & 6.93 & 6.61 & 6.37 \\
\hline $\mathrm{G}$ & - & - & 7.03 & 0.42 \\
\hline
\end{tabular}

fact that the CCA end including the attached amino acid is very likely to be flexible, but we simply start to look at the limit case, which leads to a decrease of $\mathrm{D}_{t}$ by $2.7 \%$. Since the observed change is much larger, we have to find a more extended conformation of the tRNA. As discussed already in the literature, a reasonable pathway towards a more extended form is an increase of the hinge angle between the two arms of the L-shaped tRNA. We have modelled such an increased hinge angle with reference to conformational energy calculations of Tung et al. (1984). The $\mathrm{D}_{t}$-value of the resulting form shown in Fig. $1 \mathrm{C}$ (corresponding to the form given by Tung et al. (1984) in their Fig. 5) is decreased by $8 \%$ with respect to the crystal form of $\mathrm{tRNA}^{\mathrm{Phe}}$, which is still not enough according to the light scattering data. A further extension requires "brute force", because molecular contacts contributing to tRNA stability have to be disrupted. We have extended the model shown in Fig. $1 \mathrm{C}$ without affecting the structure of the amino acid acceptor stem and the anticodon stem; we simply increased their distance by unwinding and elongation of the T- and the D-stem. During this procedure many base pairs were separated. The resulting model shown in Fig. 1D, which has been generated without a detailed check for optimal stereochemistry, is consistent with the change of the translational diffusion coefficient observed by Potts et al. (1981). However, it does not appear to be reasonable, because the transition to this extended form would require dissociation of many base pairs. In addition, the evidence accumulated in the literature (Crothers and Cole 1978) does not support any large disruption of the tRNA tertiary and/or secondary structure upon aminoacylation.

Obviously the hinge angle between the two arms of the L-shaped tRNA may also be decreased. In our simulations of such conformations we followed again the model calculations of Tung et al. (1984). Our bead model shown in Fig. $1 \mathrm{E}$ corresponds to the form given in their Fig. 6 with a bead added to represent the amino acid. A more unfolded variant of the latter model is represented in Fig. $1 \mathrm{~F}$ (without detailed check for optimal stereochemistry; coordinates of our bead models are available upon request). None of these models can be used to explain the experimental results reported by Potts et al. (1981). We have also checked, whether the relative changes may be increased by assuming a different conformation for the free tRNA. However, a decrease of the L-hinge angle, for example, leads to a slight decrease of the diffusion coefficient for the free tRNA (model G, Table 1).

The absolute values of our simulated diffusion coefficients would be changed to some extent by admitting flexibility of e.g. the CCA-end. However, the relative changes are not expected to be affected very much by such flexibility. 
In summary, we conclude that the change of the translational diffusion coefficient of tRNA ${ }^{\text {Phe }}$ upon aminoacylation reported by Potts et al. (1981) cannot be explained by an intramolecular conformation change, but must be mainly due to other processes. We suspect that the change of the diffusion coefficient is mainly due to some intermolecular association reaction. Potts et al. (1981) argued against this interpretation, but it is well known that $\mathrm{tRNA}^{\mathrm{Phc}}$ has a tendency to associate (Labuda and Porschke 1983), which should lead to some aggregates at the rather high concentrations used in their experiments. A more sensitive test for conformation changes upon aminoacylation should be possible by measurements of rotational diffusion. Whatever experimental data will arise, obviously bead model simulations will be very useful for their quantitative interpretation.

Acknowledgement. For our simulations we used the facilities of the Gesellschaft für wissenschaftliche Datenverarbeitung $\mathrm{mbH}$, Göttingen.

\section{References}

Brenner H (1967) Coupling between the translational and rotational brownian motions of rigid particles of arbitrary shape. J Colloid Interface Sci 23:407-436

Cech TR (1987) The chemistry of self-splicing RNA and RNA enzymes. Science 236:1532-1539
Crothers DM, Cole PE (1978) Conformation changes on tRNA. In: Altman S (ed) Transfer RNA. MIT Press, Cambridge, MA, pp 196-247

Davanloo P, Sprinzl M, Cramer F (1979) Proton nuclear magnetic resonance of minor nucleosides in yeast phenylalanine transfer ribonucleic acid. Conformation changes as a consequence of aminoacylation, removal of the $Y$ base, and codonanticodon interaction. Biochemistry 15:3189-3199

Garcia de la Torre J, Bloomfield VA (1981) Hydrodynamic properties of complex, rigid, biological macromolecules: theory and applications. Q Rev Biophys 14:81-139

Harvey SC, Garcia de la Torre J (1980) Coordinate systems for modeling the hydrodynamic resistance and diffusion coefficients of irregularly shaped rigid macromolecules. Macromolecules 13:960-964

Labuda D, Porschke D (1983) Codon-induced transfer RNA association. A property of transfer RNA involved in its adaptor function? J Mol Biol 167:205-209

Ladner JE, Jack A, Robertus JD, Brown RS, Rhodes D, Clark $\mathrm{BFC}$, Klug A (1975) Atomic co-ordinates for yeast phenylalanine tRNA. Nucleic Acids Res 2:1629-1637

Potts R, Fournier MJ, Ford NC Jr (1977) Effect of aminoacylation on the conformation of yeast phenylalanine tRNA. Nature 268:563-564

Potts RO, Ford NC Jr, Fournier MJ (1981) Changes in the solution structure of yeast phenylalanine transfer ribonucleic acid associated with aminoacylation and magnesium binding. Biochemistry 20:1653-1659

Quigley GJ, Seeman NC, Wang AH-J, Suddath FL, Rich A (1975) Yeast phenylalanine transfer RNA: atomic coordinates and torsion angles. Nucleic Acids Res 2:2329-2341

Tung CS, Harvey SC, McCammon JA (1984) Large-amplitude bending motions in phenylalanine transfer RNA. Biopolymers 23:2173-2193 